\title{
Structural analysis of three novel trisaccharides isolated from the fermented beverage of plant extracts
} Hideki Okada1, Eri Fukushi², Akira Yamamori1, Naoki Kawazoe ${ }^{1}$, Shuichi Onodera ${ }^{3}$, Jun Kawabata ${ }^{2}$ and Norio Shiomi*3

\begin{abstract}
Address: ${ }^{1}$ Ohtakakohso, Co, Ltd Otaru 047-0193, Japan, ${ }^{2}$ Graduate School of Agriculture, Hokkaido University, Sapporo 060-8589, Japan and ${ }^{3}$ Department of Food and Nutrition Sciences, Graduate School of Dairy Science Research, Rakuno Gakuen University, Ebetsu 069-8501, Japan

Email: Hideki Okada - oelab@ohtakakohso.co.jp; Eri Fukushi - feria@cen.agr.hokudai.ac.jp; Akira Yamamori - oelab-akira@ohtakakohso.co.jp; Naoki Kawazoe - date-lab@ohtakakohso.co.jp; Shuichi Onodera - sdera@rakuno.ac.jp; Jun Kawabata - junk@chem.agr.hokudai.ac.jp; Norio Shiomi* - n-shiomi@rakuno.ac.jp

* Corresponding author
\end{abstract}

Published: 16 July 2009

Chemistry Central Journal 2009, 3:8 doi:10.1186/1752-153X-3-8

This article is available from: http://journal.chemistrycentral.com/content/3/1/8

(c) 2009 Okada et al
Received: 25 March 2009

Accepted: 16 July 2009

\begin{abstract}
Background: A fermented beverage of plant extracts was prepared from about fifty kinds of vegetables and fruits. Natural fermentation was carried out mainly by lactic acid bacteria (Leuconostoc spp.) and yeast (Zygosaccharomyces spp. and Pichia spp.). We have previously examined the preparation of novel four trisaccharides from the beverage: $O-\beta$-D-fructopyranosyl-(2->6)-O$\beta$-D-glucopyranosyl-(I->3)-D-glucopyranose, $\quad 0$ - $\beta$-D-fructopyranosyl-(2->6)-O-[ $\beta$-Dglucopyranosyl-(I->3)]-D-glucopyranose, $\quad O-\beta$-D-glucopyranosyl-(I->I)-O- $\beta$-D-fructofuranosyl$(2<->I)-\alpha-D$-glucopyranoside and $O-\beta-D$-galactopyranosyl-(I->I)-O- $\beta$-D-fructofuranosyI-( $2<->I)-$ $\alpha$-D-glucopyranoside.

Results: Three further novel oligosaccharides have been found from this beverage and isolated from the beverage using carbon-Celite column chromatography and preparative high performance liquid chromatography. Structural confirmation of the saccharides was provided by methylation analysis, MALDI-TOF-MS and NMR measurements.

Conclusion: The following novel trisaccharides were identified: $O-\beta$-D-fructofuranosyl-(2->I)-O[ $\beta$-D-glucopyranosyl-(I->3)]- $\beta$-D-glucopyranoside (named $\quad$ "3G- $\beta$-D-glucopyranosyl $\quad \beta, \quad \beta$ isosucrose"), $O-\beta$-D-glucopyranosyl-(I->2)-O-[ $\beta$-D-glucopyranosyl-(I->4)]-D-glucopyranose (4I$\beta$-D-glucopyranosyl sophorose) and 0 - $\beta$-D-fructofuranosyl-(2->6)-O- $\beta$-D-glucopyranosyl-(I->3)D-glucopyranose (62- $\beta$-D-fructofuranosyl laminaribiose).
\end{abstract}

\section{Background}

A beverage was produced by fermentation of an extract from 50 kinds of fruits and vegetables (see Additional file 1) $[1,2]$. The extract was obtained using sucrose-osmotic pressure in a cedar barrel for seven days and was fermented by lactic acid bacteria (Leuconostoc spp.) and yeast (Zygosaccharomyces spp. and Pichia spp.) for 180 days. The fermented beverage showed scavenging activity against 1,1'-diphenyl-2-picrylhydrazyl (DPPH) radicals, and significantly reduced the ethanol-induced damage of gastric mucosa in rats [1]. Analysis by high performance anion exchange chromatography (HPAEC) showed that this beverage contained high levels of saccharides, estimated between 550 and $590 \mathrm{~g} / \mathrm{L}$; mainly glucose and fructose, and a small amount of undetermined oligosaccharides. Recently, it was reported that different positions of glyco- 
sidic linkage of oligosaccharide isomers affected physiological properties as well as physical properties [3-5]. Development of HPLC analysis with high sensitivity and separation ability enables the detection and isolation of oligosaccharides in the fermented beverage.

We have previously examined the preparation of saccharides of the fructopyranoside series from the fermented beverage of plant extracts, such as $O-\beta$-D-fructopyranosyl(2->6)-D-glucopyranose [2], O- $\beta$-D-fructopyranosyl-(2$>6)-O-\beta$-D-glucopyranosyl-(1->3)-D-glucopyranose and $O-\beta$-D-fructopyranosyl-(2->6)-O-[ $\beta$-D-glucopyranosyl(1->3)]-D-glucopyranose [6]. The characteristics of $O-\beta$ D-fructopyranosyl-(2->6)-D-glucopyranose were non-cariogenicity and low digestibility, and the unfavorable bacteria that produce mutagenic substances did not use the saccharide $[7,8]$. Recently, we have studied isolation and identification of novel non-reducing trisaccharides, such as $O$ - $\beta$-D-glucopyranosyl-(1->1)-O- $\beta$-D-fructofuranosyl$(2<->1)-\alpha-\mathrm{D}$-glucopyranoside and $O-\beta$-D-galactopyranosyl-(1->1)-O- $\beta$-D-fructofuranosyl-(2<->1)- $\quad \alpha$-D-glucopyranoside from the beverage [9], and those saccharides were confirmed to be produced by fermentation.

In this paper, we have confirmed structures of the novel trisaccharides (Fig. 1): O- $\beta$-D-fructofuranosyl-(2->1)-O[ $\beta$-D-glucopyranosyl-(1->3)]- $\beta$-D-glucopyranoside (named "3 ${ }^{\mathrm{G}}-\beta$-D-glucopyranosyl $\beta, \beta$-isosucrose"), $O-\beta$ D-glucopyranosyl-(1->2)-O-[ $\beta$-D-glucopyranosyl-(1$>4)$ ]-D-glucopyranose (41- $\beta$-D-glucopyranosyl sophorose) and $O-\beta$-D-fructofuranosyl-(2->6)-O- $\beta$-D-glucopyranosyl-(1->3)-D-glucopyranose (62- $\beta-\mathrm{D}-$ fructofuranosyl laminaribiose), isolated from the fermented beverage using methylation analysis, MALDITOF-MS and NMR measurements.

\section{Results and discussion}

Saccharides 1, 2 and 3 were isolated from the fermented beverage of plant extracts using carbon-Celite column chromatography, and were shown to be homogeneous using anion exchange HPLC $\left[t_{\mathrm{R} \text {, sucrose }}\right.$ (relative retention time; retention time of sucrose = 1.0): 1.89, 2.23 and 2.40 respectively]. The retention time of saccharides 1, 2 and 3 did not correspond to that of any authentic saccharides Iglucose (0.62), fructose (0.68), sucrose (1.00), maltose (1.43), trehalose (0.58), laminaribiose (1.33), raffinose (1.23), 1-kestose (1.47), 6-kestose (1.75), neokestose (1.90), maltotriose (2.59), panose (1.87), nystose (2.06), fructosylnystose (3.81), O- $\beta$-D-fructopyranosyl-(2->6)-Dglucopyranose (0.83) [2], O- $\beta$-D-fructopyranosyl-(2->6)$O-\beta$-D-glucopyranosyl-(1->3)-D-glucopyranose (1.74) $[6], \quad O-\beta$-D-fructopyranosyl-(2->6)-O-[ $\beta$-D-glucopyranosyl-(1->3)]-D-glucopyranose (1.72) [6], O- $\beta$-D-glucopyranosyl-(1->1)-O- $\beta$-D-fructofuranosyl-(2<->1)- $\quad \alpha$-Dglucopyranoside (1.24) [9], O- $\beta$-D-galactopyranosyl-(1-
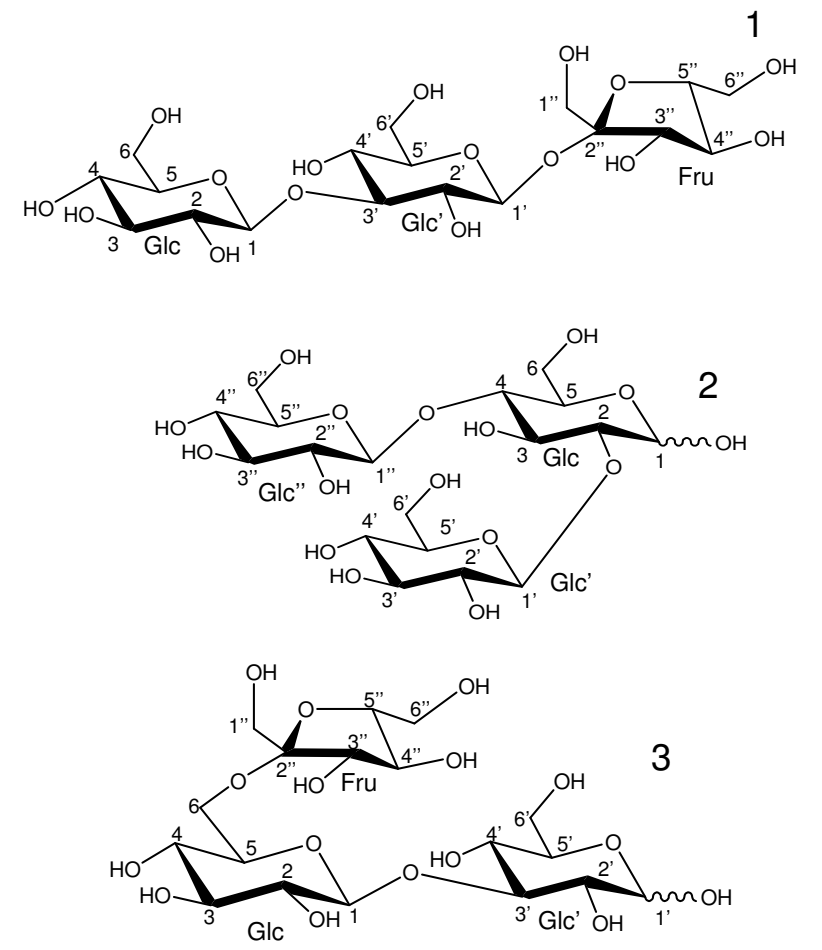

Figure I

Structures of $O-\beta-D$-fructofuranosyl-(2->I)-O-[ $\beta$-Dglucopyranosyl-(I->3)]- $\beta$-D-glucopyranoside (I), O- $\beta$ D-glucopyranosyl-(I->2)- O-[ $\beta$-D-glucopyranosyl-(I$>4)$ ]-D-glucopyranose (2) and O- $\beta$-D-fructofuranosyl(2->6)- O- $\beta$-D-glucopyranosyl-(I->3)-D-glucopyranose (3).

$>1)$-O- $\beta$-D-fructofuranosyl-(2<->1)- $\alpha$-D-glucopyranoside (0.84) [9], 2(2- $\alpha$-D-glucopyranosyl)isokestose (1.57) [10], 2(2- $\alpha$-D-glucopyranosyl) $)_{2}$ isokestose (1.79) [10], 2(2- $\alpha$-D-glucopyranosyl) ${ }_{3}$ isokestose (2.09) [10], 2(2- $\alpha$ D-glucopyranosyl)nystose (2.17) [10], 2(2- $\alpha$-D-glucopyranosyl) ${ }_{2}$ nystose (2.63) [10], O- $\alpha$-D-glucopyranosyl-(1$>2$ )-O- $\alpha$-D-xylopyranosyl-(1->2)- $\beta$-D-fructofuranoside (1.51) [11], O- $\alpha$-D-glucopyranosyl-(1->2)-O- $\alpha$-D-glucopyranosyl-(1->2)-O- $\alpha$-D-xylopyranosyl-(1->2)- $\beta$-D-fructofuranoside (1.80) [11].

The degree of polymerization of saccharides 1, 2 and 3 was established as 3 by measurements of $[\mathrm{M}+\mathrm{Na}]$ ions $(\mathrm{m} /$ $z$ : 527) using TOF-MS (see Fig. 2), and analysis of the molar ratios of D-glucose to D-fructose in the acid hydrolysates. Acid hydrolysates of saccharides $\mathbf{1}$ and $\mathbf{3}$ were liberated to glucose and fructose, and saccharide 2 was liberated to glucose. From the GC analysis, relative retention times of the methanolysate of the permethylated saccharides were investigated $\left[t_{R}\right.$ (relative retention time; retention time of methyl 2, 3, 4, 6-tetra-O-methyl- $\beta$-Dglucoside $=1.0$; retention time, $9.60 \mathrm{~min})]$. The meth- 

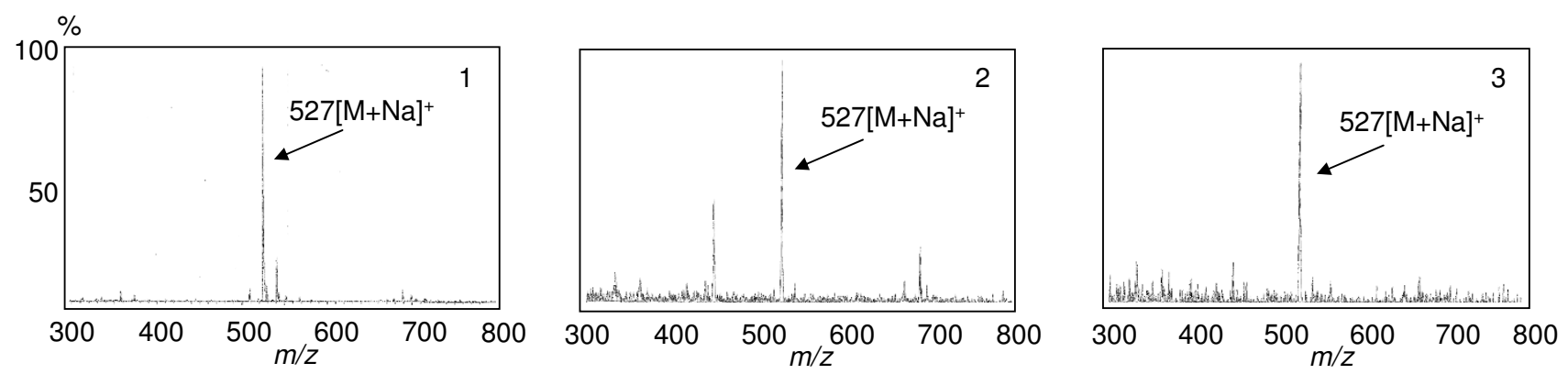

Figure 2

MALDI-TOF-MS spectra of saccharides I, 2, and 3. I: saccharide I, 2: saccharide 2, 3: saccharide 3.

anolysate of permethylated saccharide 1 exhibited six peaks (see Additional file 2) corresponding to methyl 2,3,4,6-tetra-O-methyl-D-glucoside $\left(\mathrm{t}_{\mathrm{R}^{\prime}}, 0.94\right.$ and 1.48), methyl 2,4,6-tri-O-methyl-D-glucoside $\left(\mathrm{t}_{\mathrm{R}^{\prime}}, 3.27\right.$ and 4.81) and methyl 1,3,4,6-tetra-O-methyl-D-fructoside $\left(\mathrm{t}_{\mathrm{R}}, 1.06\right.$ and 1.32). The methanolysate of permethylated saccharide 3 also exhibited six peaks (see Additional file 2) corresponding to methyl 2,3,4-tri-O-methyl-D-glucoside $\left(\mathrm{t}_{\mathrm{R}}\right.$ 2.58 and 3.59), methyl 2,4,6-tri-O-methyl-D-glucoside $\left(\mathrm{t}_{\mathrm{R}}, 3.22\right.$ and 4.73), and methyl 1,3,4,6-tetra-O-methyl-Dfructoside $\left(t_{R}, 1.07\right.$ and 1.29). On the other hand, the methanolysate of permethylated saccharide 2 exhibited two peaks (see Additional file 2) corresponding to methyl 2,3,4,6-tetra-O-methyl-D-glucoside ( $\mathrm{t}_{\mathrm{R}^{\prime}} 0.97$ and 1.47). GC-MS analysis on the retention times and fragmentation patterns of the methyl glucosides [12] showed the two peaks (10.08 $\mathrm{min}$ and $10.21 \mathrm{~min}$ ) from the methanolysate of permethylated saccharide 2 to be methyl 3,6di-O-methyl-D-glucoside. From these findings above, saccharides 1, 2 and 3 were proved to be, $O$-D-fructofuranosyl-(2->1)-O-[D-glucopyranosyl-(1->3)]-D-

glucopyranoside, O-D-glucopyranosyl-(1->2)-O-[D-glucopyranosyl-(1->4)]-D-glucose and O-D-fructofuranosyl(2->6)-O-D-glucopyranosyl-(1->3)-D-glucose, respectively.

The structural confirmations of saccharides 1, 2 and 3 according to ${ }^{1} \mathrm{H}$ and ${ }^{13} \mathrm{C}$ NMR analyses and the subsequent complete assignment of ${ }^{1} \mathrm{H}$ and ${ }^{13} \mathrm{C}$ NMR signals of the three saccharides were carried out using 2D-NMR techniques.

First, the NMR spectra of saccharide 1 were analyzed. The HSQC-TOCSY spectrum revealed the ${ }^{1} \mathrm{H}$ and ${ }^{13} \mathrm{C}$ signals of each Glc, Glc' and Fru. The isolated methylene was assigned as $\mathrm{H}-1$ and $\mathrm{C}-1$ in Fru. The other three methylene carbons were assigned as C- 6 in these residues. The COSY spectrum assigned the spin systems of these residues; from H-1 to H-3 and H-1' to H-3' (Fig. 3(a)), and from H-3" to
H-6". The corresponding ${ }^{13} \mathrm{C}$ signals were assigned by HSQC spectrum (Fig. 3(b)). These results clarified the assignment of ${ }^{1} \mathrm{H}$ and ${ }^{13} \mathrm{C}$ NMR signals of each residue. The position of the glucosidic linkage and fructosidic linkage was analyzed as follows. The C-3' showed the HMBC $[13,14]$ correlations between H-1 (Fig. 3(c)). The J (H-1/ $\mathrm{H}-2)$ value was $7.9 \mathrm{~Hz}$. These results indicated the Glc $1 \beta$ ->3' Glc linkage, namely the laminaribiose moiety. The C2 " showed the HMBC correlations to H-1'. The J $\left(\mathrm{H}-1{ }^{\prime} / \mathrm{H}-\right.$ $\left.2^{\prime}\right)$ value was $7.4 \mathrm{~Hz}$. These results indicated the Glc' $1 \beta$ $>2 \beta$ Fru linkage, and all ${ }^{1} \mathrm{H}$ and ${ }^{13} \mathrm{C}$ NMR signals were assigned as shown in Additional file 3.

The coupling patterns of overlapped ${ }^{1} \mathrm{H}$ were analyzed by the SPT method $[15,16]$. Due to strong coupling between $\mathrm{H}-4$ ' and H-5', these couplings could not be analyzed in first order.

The NMR spectra of saccharide 2 showed that it was an anomeric mixture at the Glc. The $\alpha$ anomer was predominant. The COSY spectrum was assigned from H-1 to H-6. The C-4 showed the HMBC correlations between H-1" (Fig. 4(a) and 4(b)). The $J(\mathrm{H}-1$ "/H-2") value was 7.6-7.8 $\mathrm{Hz}$. These results indicated the Glc" $1 \beta->4$ Glc linkage, namely the cellobiose moiety. The $\mathrm{C}-2$ showed the HMBC correlations to $\mathrm{H}-1^{\prime}$. The $J\left(\mathrm{H}-1^{\prime} / \mathrm{H}-2^{\prime}\right)$ value was $7.6 \mathrm{~Hz}$. These results indicated the Glc' $1 \beta->2$ Glc linkage, and all ${ }^{1} \mathrm{H}$ and ${ }^{13} \mathrm{C}$ NMR signals were assigned as shown in Additional file 3 .

The NMR spectra of saccharide 3 were analyzed in the same manner as those of saccharide 2 . Saccharide 3 was also an anomeric mixture at the Glc'. The $\beta$ anomer was predominant. The HSQC-TOCSY spectrum revealed the ${ }^{1} \mathrm{H}$ and ${ }^{13} \mathrm{C}$ signals of each Glc, Glc' and Fru. The isolated methylene was assigned as $\mathrm{H}-1$ " and $\mathrm{C}-1$ ". The other three methylene carbons were assigned as C- 6 in these residues (Fig. 5(a)). The position of the glucosidic linkage and fructosidic linkage was analyzed as follows. The C-3' 
(a)

(b)

(c)

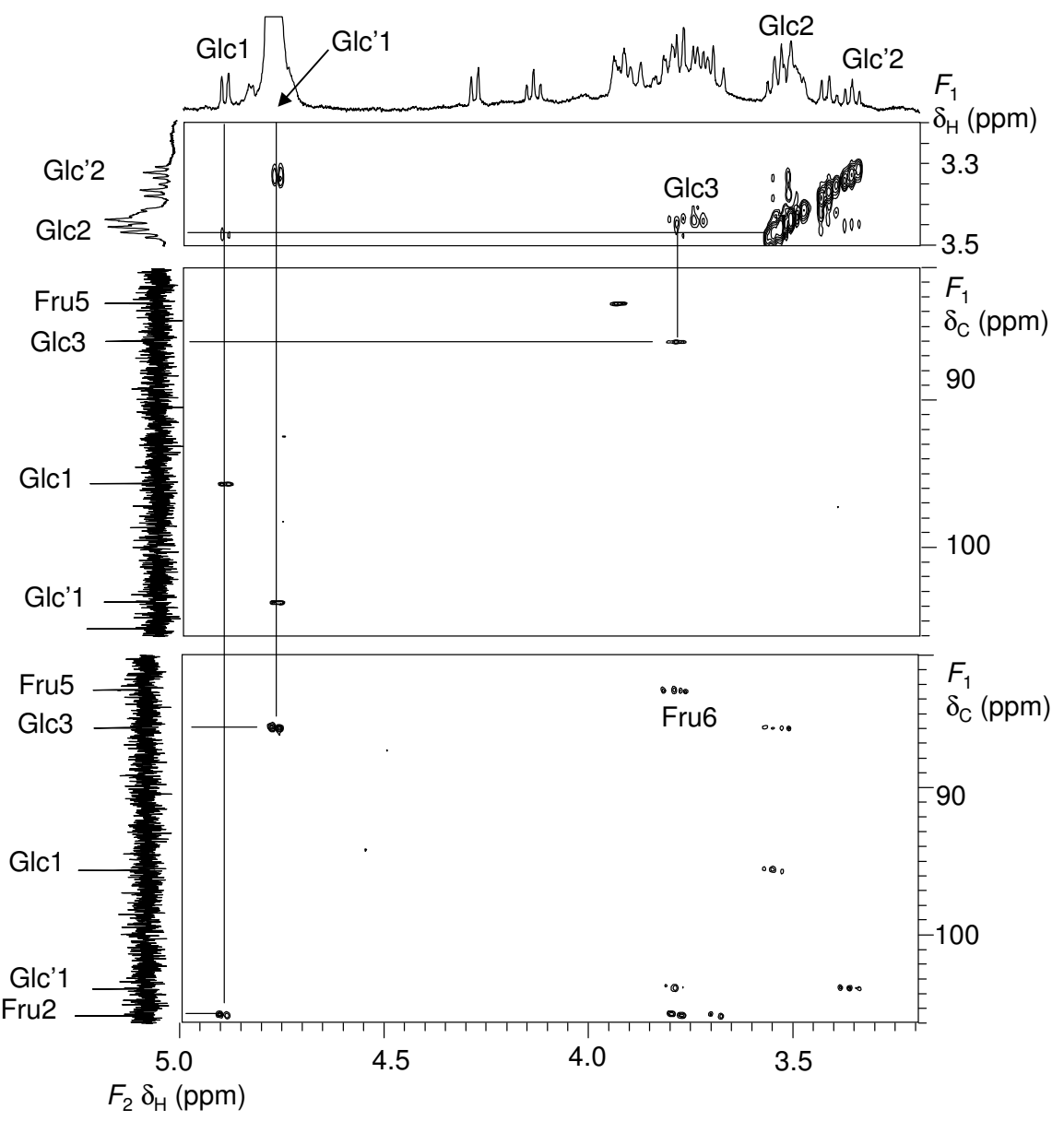

Figure 3

Part of COSY (a), HSQC (b) and HMBC (c) spectra of saccharide I.

showed the HMBC correlations between H-1 (Fig. 5(b)). The J (H-1/H-2) value was 7.9 Hz. These results indicated the Glc $1 \beta \rightarrow 3$ Glc' linkage, namely the laminaribiose moiety. The C-2 showed the HMBC correlations to H-6. These results indicated the Glc $6<-2 \beta$ Fru linkage, and all ${ }^{1} \mathrm{H}$ and ${ }^{13} \mathrm{C}$ NMR signals were assigned as shown in Additional file 3 .

From all of these findings, saccharides 1, 2, and 3 from the fermented beverage of plant extracts were confirmed to be new oligosaccharides (Fig. 1):O- $\beta$-D-fructofuranosyl-(2$>1)$-O-[ $\beta$-D-glucopyranosyl-(1->3)]- $\beta$-D-glucopyranoside (named "3G- $\beta$-D-glucopyranosyl $\beta$, $\beta$-isosucrose"), $O$ - $\beta$-D-glucopyranosyl-(1->2)-O-[ $\beta$-D-glucopyranosyl-(1$>4)$ ]-D-glucopyranose (41- $\beta$-D-glucopyranosyl sophorose) and $O-\beta$-D-fructofuranosyl-(2->6)-O- $\beta$-D-glucopyranosyl-(1->3)-D-glucopyranose fructofuranosyl laminaribiose).
Synthesis of the saccharides by fermentation of plant extracts was investigated using HPAEC. Almost all of the monosaccharides were removed from the fermented and unfermented beverages of plant extracts by the batch method with Charcoal. The saccharides 1, 2, and 3 were observed in the fermented beverage, but were not present in the unfermented one. Therefore, saccharides 1, 2, and 3 were confirmed to have been produced during fermentation of the beverage of plant extracts (Fig. 6).

\section{Conclusion}

We have previously found that the fermented beverage contained the novel saccharide, $O-\beta$-D-fructopyranosyl(2->6)-D-glucopyranose, which is produced by fermentation. The saccharide showed low digestibility. The saccharide was selectively used by beneficial bacteria, Bifidobacterium adolescentis and B. longum, but was not used by unfavorable bacteria, Clostridium perfringens, Escherichia coli and Enterococcus faecalis that produce muta- 
(a)

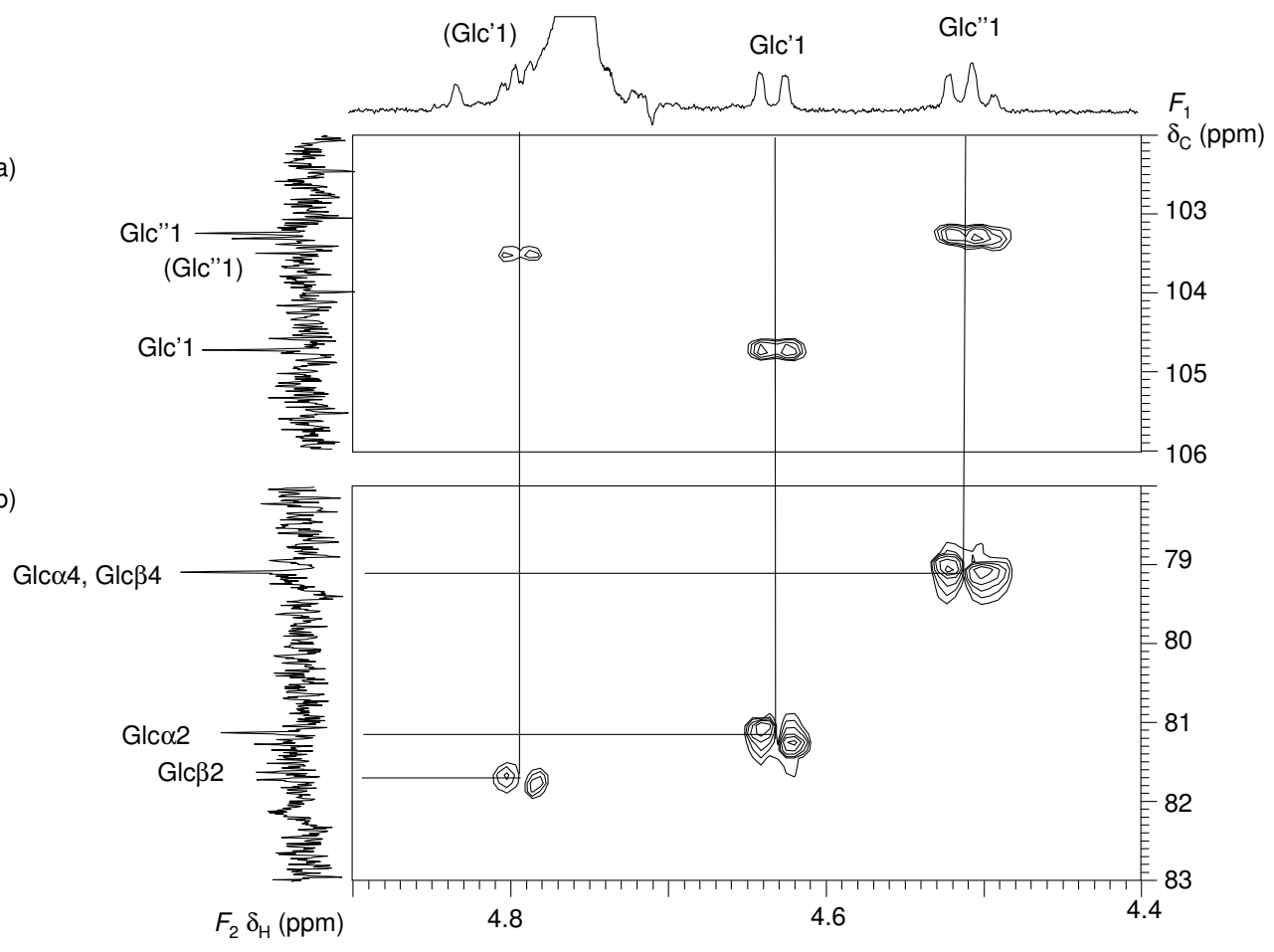

Figure 4

Part of HSQC (a) and HMBC (b) spectra of saccharide 2. ( ) = minor anomer.

genic substances [8]. It is interesting to study the biological functions of other oligosaccharides existing in the beverage. In this report, three novel oligosaccharides have been found from this beverage, and isolated from the beverage using carbon-Celite column chromatography and preparative high performance liquid chromatography. Structural confirmation of the saccharides was provided by methylation analysis, MALDI-TOF-MS and NMR measurements. These saccharides were identified as new trisaccharides:O- $\beta$-D-fructofuranosyl-(2->1)-O-[ $\beta-\mathrm{D}-$

glucopyranosyl-(1->3)]- $\beta$-D-glucopyranoside (named "3G- $\beta$-D-glucopyranosyl $\beta$, $\beta$-isosucrose" ), O- $\beta$-D-glucopyranosyl-(1->2)-O-[ $\beta$-D-glucopyranosyl-(1->4)]-D-glucopyranose ( $4^{1}-\beta$-D-glucopyranosyl sophorose) and $O-\beta$ D-fructofuranosyl-(2->6)-O- $\beta$-D-glucopyranosyl-(1->3)D-glucopyranose ( $6^{2}-\beta$-D-fructofuranosyl laminaribiose). These saccharides were confirmed to be produced during fermentation.

\section{Experimental}

Preparation of fermented beverage of plant extract

For preparation of the initial juice, 50 kinds of fruits and vegetables were used to produce the final extract as shown in a previous paper $[1,2]$. The 50 fruits and vegetables were cut, sliced or diced into small pieces, mixed and put in cedar barrels. Afterwards, an equivalent weight of sucrose was added to the samples, mixed well to allow high contact between the samples and sucrose, and then the barrels were left for one week at room temperature. The juice exudate was then separated without compression from solids and used for fermentation. The fermented beverage was obtained by incubation of the juice at $37^{\circ} \mathrm{C}$ in the dark by natural fermentation using yeast (Zygosaccharomyces spp. and Pichia spp.) and lactic acid bacteria (Leuconostoc spp.). After 7 days, the fermented beverage was kept in a closed enameled tank at $37^{\circ} \mathrm{C}$ for 180 days for additional maturation and ageing, finally obtaining a brown and slightly sticky liquid.

\section{High performance anion-exchange chromatography (HPAEC)}

The oligosaccharides were analyzed using a Dionex Bio LC Series apparatus equipped with an HPLC carbohydrate column (Carbo Pack PA1, inert styrene divinyl benzene polymer) and pulsed amperometric detection (PAD) $[17,18]$. The mobile phase consisted of eluent A (150 mM $\mathrm{NaOH}$ ) and eluent B (500 mM sodium acetate in $150 \mathrm{mM}$ $\mathrm{NaOH}$ ) with a sodium acetate gradient as follows: $0-1$ min, $25 \mathrm{mM}$; 1-2 min, 25-50 mM; 2-20 min, 50-200 $\mathrm{mM}$; 20-22 $\min , 500 \mathrm{mM}$; 22-30 min, $25 \mathrm{mM}$; using a flow rate through the column of $1.0 \mathrm{~mL} / \mathrm{min}$. The applied PAD potentials for E1 (500 ms), E2 (100 ms), and E3 (50 


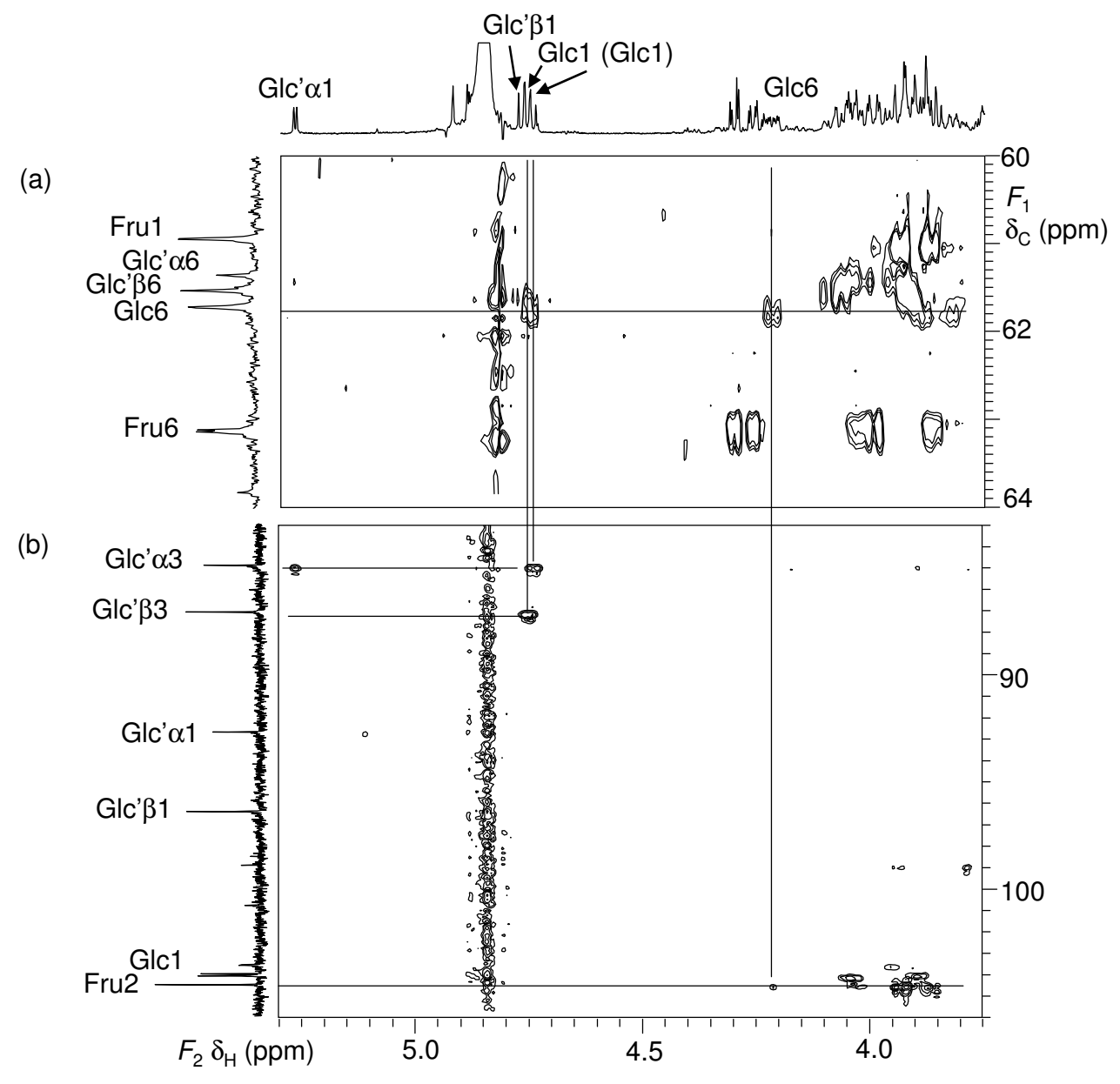

Figure 5

Part of HSQC-TOCSY (a) and HMBC (b) spectra of saccharide 3. ( ) = minor anomer.

ms) were $0.1,0.6$, and $-0.6 \mathrm{~V}$ respectively, and the output range was $1 \mu \mathrm{C}$.

\section{Isolation of saccharides}

The fermented beverage of plant extracts (1000 g) was loaded onto a carbon-Celite $[1: 1$; charcoal (Wako Pure Chemical Industries, Ltd; Osaka, Japan) and Celite-535 (Nacalai Tesque Inc, Osaka, Japan)] column (4.5 × 35 $\mathrm{cm})$, and was successively eluted with water $(14 \mathrm{~L}), 5 \%$ ethanol (30 L) and 30\% ethanol (10 L). Almost all of the glucose and fructose were eluted with water $(4 \mathrm{~L})$, and then saccharides 1, 2 and 3 were eluted with $30 \%$ ethanol (1-2 L). The 30\% ethanol fraction containing saccharides 1, 2 and 3 was concentrated in vacuo and freeze-dried to give $894 \mathrm{mg}$ of sample. Subsequently, the 30\% ethanol fraction was successfully repeatedly purified using an HPLC system (Tosoh, Tokyo, Japan) equipped with an Amide- 80 column $(7.8 \mathrm{~mm} \times 30 \mathrm{~cm}$, Tosoh, Tokyo, Japan) at $80^{\circ} \mathrm{C}$, and eluted with $80 \%$ acetonitrile at 2.0
$\mathrm{mL} / \mathrm{min}$, and using refractive index detection. Furthermore, the saccharides were purified by HPLC with the ODS-100 V column $(4.6 \mathrm{~mm} \times 25 \mathrm{~cm}$, Tosoh, Tokyo, Japan) at room temperature, and eluted with water at 0.5 $\mathrm{mL} / \mathrm{min}$. Purified saccharides 1 (2.5 mg), 2 (2.2 mg) and $3(2.0 \mathrm{mg})$ were obtained as white powders.

\section{Methylation and methanolysis}

Methylation of the oligosaccharides was carried out by the method of Hakomori [19]. The permethylated saccharides were methanolyzed by heating with $1.5 \%$ methanolic hydrochloric acid at $96^{\circ} \mathrm{C}$ for 10 or $180 \mathrm{~min}$. The reaction mixture was treated with Amberlite IRA-410 $\left(\mathrm{OH}^{-}\right)$to remove hydrochloric acid, and evaporated in vacuo to dryness. The resulting methanolysate was dissolved in a small volume of methanol and analyzed using gas liquid chromatography. 


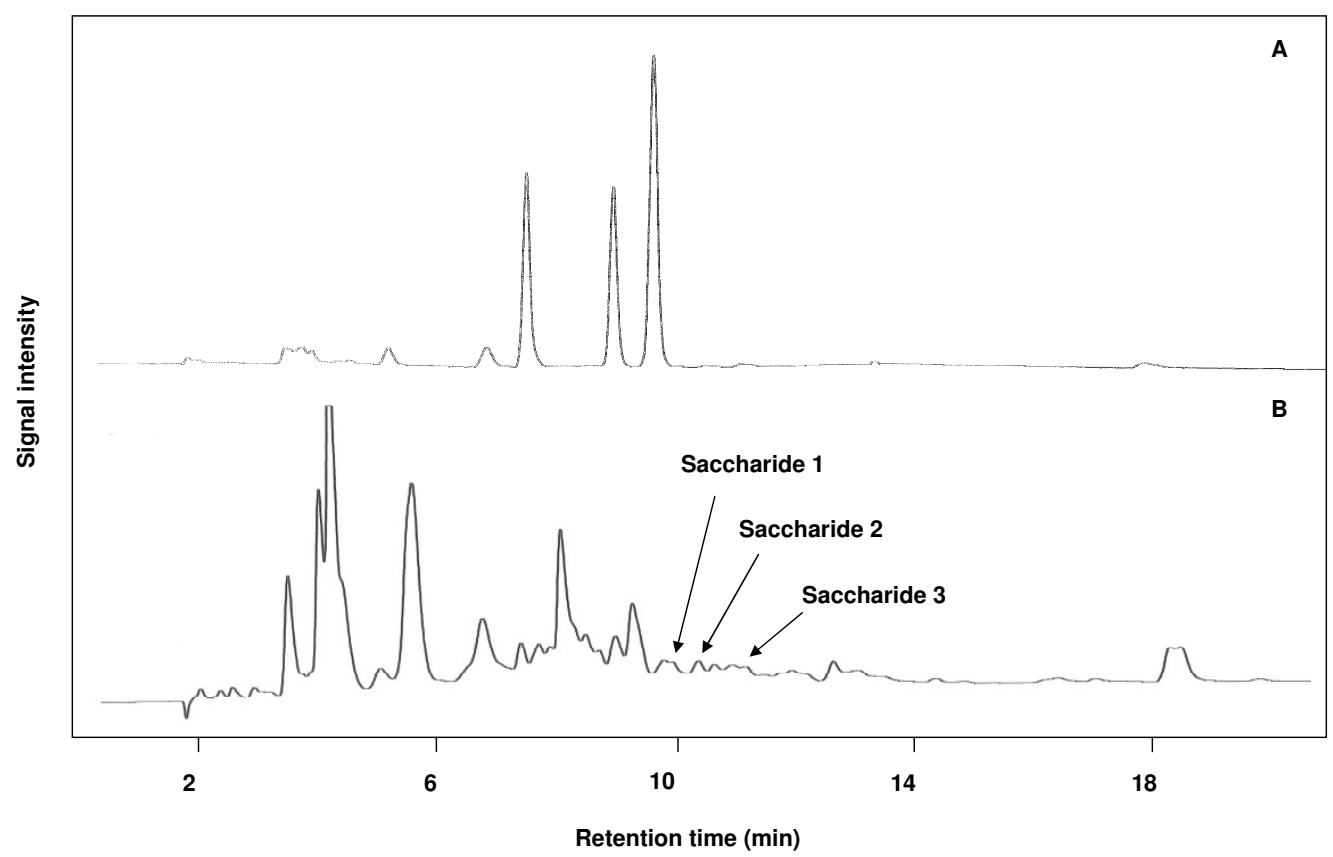

\section{Figure 6}

High performance liquid chromatogram of fermentation products. A: The beverage of plant extract was fermented for 0 days. B: The beverage of plant extract was fermented for 180 days. The beverage $(100 \mathrm{~mL})$ fermented for 0 or 180 days was mixed with charcoal ( $10 \mathrm{~g})$, stirred for $3 \mathrm{~h}$. and filtered. The charcoal was extracted with $30 \%$ ethanol $(500 \mathrm{~mL})$ three times. The ethanol extracts were combined, concentrated to dryness and solubilized with one $\mathrm{mL}$ of distilled water. The sugar solution was analyzed by HPAEC.

\section{Gas liquid chromatography (GLC)}

For the analysis of the methanolysate, GLC was carried out using a Shimadzu GC-8A gas chromatograph equipped with a glass column $(2.6 \mathrm{~mm} \times 2 \mathrm{~m})$ packed with $15 \%$ butane 1,4 -diol succinate polyester on acidwashed Celite at $175^{\circ} \mathrm{C}$. Flow rate of the nitrogen gas carrier was $40 \mathrm{~mL} / \mathrm{min}$.

\section{GC-MS analysis}

GC-MS analysis was performed using a JMS-AX500 mass spectrometer (JEOL, Japan) using a DB-17HT capillary column (30 $\mathrm{m} \times 0.25 \mathrm{~mm}$ I.D., J \& W Scientific, USA). Injection temperature was $200^{\circ} \mathrm{C}$. The column temperature was kept at $50^{\circ} \mathrm{C}$ for 2 min after sample injection, increased to $150^{\circ} \mathrm{C}$ at $50^{\circ} \mathrm{C} / \mathrm{min}$, kept at $150^{\circ} \mathrm{C}$ for $1 \mathrm{~min}$, and then increased to $250^{\circ} \mathrm{C}$ at $4^{\circ} \mathrm{C} / \mathrm{min}$. The mass spectra were recorded in the electron ionization (EI) mode.

\section{MALDI-TOF-MS}

MALDI-TOF-MS spectra were measured using a Shimadzu-Kratos mass spectrometer (KOMPACT Probe) in positive ion mode with $2.5 \%$-dihydroxybenzoic acid as a matrix. Ions were formed by a pulsed UV laser beam (nitrogen laser, $337 \mathrm{~nm}$ ). Calibration was done using 1kestose as an external standard.

\section{NMR measurement}

The saccharide (ca. $2 \mathrm{mg}$ ) was dissolved in $0.06 \mathrm{~mL}$ (saccharide 1) and $0.4 \mathrm{~mL}$ (saccharide 2 and 3) $\mathrm{D}_{2} \mathrm{O}$. NMR spectra were recorded at $27^{\circ} \mathrm{C}$ with a Bruker AMX 500 spectrometer ( $1 \mathrm{H} 500 \mathrm{MHz}, 13 \mathrm{C} 125 \mathrm{MHz}$ ) equipped with a $2.5 \mathrm{~mm} \mathrm{C} / \mathrm{H}$ dual probe (saccharide 1 ), a $5 \mathrm{~mm}$ diameter $\mathrm{C} / \mathrm{H}$ dual probe (1D spectra of saccharide 2 and $3)$, and a $5 \mathrm{~mm}$ diameter TXI probe (2D spectra of saccharide 2 and 3$)$. Chemical shifts of ${ }^{1} \mathrm{H}\left(\delta_{\mathrm{H}}\right)$ and ${ }^{13} \mathrm{C}\left(\delta_{\mathrm{C}}\right)$ in ppm were determined relative to an external standard of sodium $\left[2,2,3,3-{ }^{2} \mathrm{H}_{4}\right]-3$-(trimethylsilyl)-propanoate in $\mathrm{D}_{2} \mathrm{O}\left(\delta_{\mathrm{H}} 0.00 \mathrm{ppm}\right)$ and 1,4 -dioxane $\left(\delta_{\mathrm{C}} 67.40 \mathrm{ppm}\right)$ in $\mathrm{D}_{2} \mathrm{O}$, respectively. ${ }^{1} \mathrm{H}-{ }^{1} \mathrm{H}$ COSY [20,21], HSQC [22], HSQC-TOCSY [22,23] $\mathrm{CH}_{2}$-selected E-HSQC-TOCSY [24], HMBC $[13,14]$ and CT-HMBC $[13,14]$ spectra were obtained using gradient selected pulse sequences. The TOCSY mixing time (0.15 s) was composed of DIPSI-2 composite pulses.

\section{Competing interests}

The authors declare that they have no competing interests.

\section{Authors' contributions}

$\mathrm{HO}, \mathrm{AY}$ and NK performed data analysis, and contributed to drafting the manuscript. EF and JK collected the NMR 
data. NS and SO conceived of the study, participated in its design and contributed to drafting the manuscript. All authors read and approved the final manuscript.

\section{Additional material}

\section{Additional file 1}

Table S1. Materials of fermented beverage of plant extract. Click here for file

[http://www.biomedcentral.com/content/supplementary/1752153X-3-8-S1.xls]

\section{Additional file 2}

Table S2. Gas-liquid chromatographic analysis of methanolysates of permethylated saccharides 1, 2 and 3.

Click here for file

[http://www.biomedcentral.com/content/supplementary/1752-

153X-3-8-S2.xls]

\section{Additional file 3}

Table S3. ${ }^{1} \mathrm{H}$ and ${ }^{13} \mathrm{C}$ NMR spectral data of saccharides 1,2 and 3. Click here for file

[http://www.biomedcentral.com/content/supplementary/1752153X-3-8-S3.xls]

\section{References}

I. Okada H, Kudoh K, Fukushi E, Onodera S, Kawabata J, Shiomi N Antioxidative activity and protective effect of fermented plant extract on ethanol-induced damage to rat gastric mucosa. J Jap Soc Nutr Food Sci 2005, 58:209-2I 5.

2. Okada H, Fukushi E, Yamamori A, Kawazoe N, Onodera S, Kawabata J, Shiomi N: Structural analysis of a novel saccharide isolated from fermented beverage of plant extract. Carbohydr Res 2006 341:925-929.

3. Kohmoto T, Fukui F, Takaku H, Machida Y, Arai M, Mitsuoka T: Effect of isomalto-oligosaccharides on human fecal flora. Bifdobacteria Microflora 1988, 7:61-69.

4. Murosaki S, Muroyama K, Yamamoto Y, Kusaka H, Liu T, Yoshikai Y: Immunopotentiating activity of nigerooligosaccharides for the Thelper I-like immune response in mice. Biosci Biotechnol Biochem 1999, 63:373-378.

5. Murosaki S, Muroyama K, Yamamoto Y, Liu T, Yoshikai Y: Nigerooligosaccharides augments natural killer activity of hepatic mononuclear cells in mice. Int Immunopharmacol 2002, 2:15I-159.

6. Kawazoe N, Okada H, Fukushi E, Yamamori A, Onodera S, Kawabata J, Shiomi N: Two novel oligosaccharides isolated from a beverage produced by fermentation of a plant extract. Carbohydr Res 2008, 343:549-554.

7. Okada H, Kawazoe N, Yamamori A, Onodera S, Shiomi N: Structural analysis and synthesis of oligosaccharides isolated from fermented beverage of plant extract. J Appl Glycosci 2008, 55: $143-148$.

8. Okada H, Kawazoe N, Yamamori A, Onodera S, Kikuchi M, Shiomi N Characteristics of O- $\beta$-D-fructopyranosyl-(2->6)-D-glucopyranose isolated from fermented beverage of plant extract. J Appl Glycosci 2008, 55: 179-182.

9. Kawazoe N, Okada H, Fukushi E, Yamamori A, Onodera S, Kawabata J, Shiomi N: Structural analysis of two trisaccharides isolated from fermented beverage of plant extract. Open Glycosci 2008, I:25-30.

10. Okada H, Fukushi E, Onodera S, Nishimato T, Kawabata J, Kikuchi M, Shiomi N: Synthesis and structural analysis of five novel oligosaccharides prepared by glucosyltransfer from $\beta$-D-glucose I-phosphate to isokestose and nystose using
Thermoanaerobacter brockii kojibiose phosphorylase. Carbohydr Res 2003, 338:879-885.

II. Takahashi N, Fukushi E, Onodera S, Benkeblia N, Nishimato T, Kawabata J, Shiomi N: Three novel oligosaccharides synthesized using Thermoanaerobacter brockii kojibiose phosphorylase. Chem Cent / 2007, I: 18

12. Funakoshi I: Mass spectrum. In Seikagaku data book Edited by: Yamashina I. Tokyo: Tokyokagakudojin; 1979:606-668.

13. Bax A, Summers MF: ' $\mathbf{H}$ and ${ }^{13} \mathbf{C}$ assignments from sensitivityenhanced detection of heteronuclear multiple-bond connectivity by 2D multiple quantum NMR. J Am Chem Soc 1986, 108:2093-2094.

14. Hurd RE, John BK: Gradient-enhanced proton-detected heteronuclear multiple-quantum coherence spectroscopy. J Magn Reson 1991, 91:648-653.

15. Pachler KGR, Wessels PL: Selective Population Inversion (SPI). A pulsed double resonance method in FT NMR spectroscopy equivalent to INDOR. J Magn Reson 1973, I 2:337-339.

16. Uzawa J, Yoshida S: A new selective population transfer experiment using a double pulsed field gradient spin-echo. Magn Reson Chem 2004, 42: 1046-1048.

17. Rocklin RD, Pohl CA: Determination of carbohydrate by anion exchange chromatography with pulse amperometric detection. J Liq Chromatogr 1983, 6:1577-1590.

18. Johnson DC: Carbohydrate detection gains potential. Nature 1986, 32 I:45I-452

19. Hakomori S: A rapid permethylation of glycolipid and polysaccharide catalyzed by methylsulfinyl carbanion in dimethylsulfoxide. J Biochem 1964, 55:205-208.

20. Aue WP, Batholdi E, Ernst RR: Two-dimensional spectroscopy. Application to nuclear magnetic resonance. J Chem Phys 1976, 64:2229-2246.

21. von Kienlin M, Moonen CTW, Toorn A von der, van $\mathrm{Zijl}$ PCM: Rapid recording of solvent-suppressed 2D COSY spectra with inherent quadrupole detection using pulsed field gradients. $J$ Magn Reson 1991, 93:423-429.

22. Willker W, Leibfritz D, Kerssebaum R, Bermel W: Gradient selection in inverse heteronuclear correlation spectroscopy. Magn Reson Chem 1993, 3 I:287-292.

23. Domke T: A new method to distinguish between direct and remote signals in proton-relayed $\mathbf{X}, \mathbf{H}$ correlations. J Magn Reson 199I, 95: I74-I77.

24. Yamamori A, Fukushi E, Onodera S, Kawabata J, Shiomi N: NMR analysis of mono- and difructosyllactosucrose synthesized by IF-fructosyltransferase purified from roots of asparagus (Asparagus officinalis L.). Magn Reson Chem 2002, 40:54 I-544.

\section{Publish with ChemistryCentral and every} scientist can read your work free of charge

"Open access provides opportunities to our colleagues in other parts of the globe, by allowing anyone to view the content free of charge."

W. Jeffery Hurst, The Hershey Company.

- available free of charge to the entire scientific community

- peer reviewed and published immediately upon acceptance

- cited in PubMed and archived on PubMed Central

- yours - you keep the copyright

Submit your manuscript here:

http://www.chemistrycentral.com/manuscript/

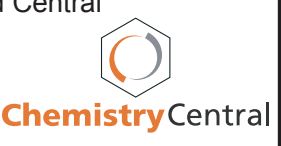

